\title{
Interactive comment on "On the alignment of velocity and magnetic fields within magnetosheath jets" by Ferdinand Plaschke et al.
}

\section{Anonymous Referee \#4}

Received and published: 3 September 2019

\section{Overview:}

Paper focuses on studying if there is statistical, observational, evidence supporting the simulations of Karimabadi et al., 2014 that magnetosheath jets make the ambient magnetic field more aligned with the jet velocity. Study uses data (FPI for ion moments and FGM for the magnetic field) from the four Magnetosphere Multi-Scale (MMS) spacecraft between September 2015 and May 2017. The main conclusions by the authors, obtained based on superposed epoch analysis of the pre and post jet angles between ion velocity and magnetic field, is that while jets generally modify the magnetic field, the alignment of the magnetic field with the jet flow is relatively small. They suspect the discrepancy may be due to the 2-D simulation geometry, while the real nature is 3-D.

Paper addresses a compelling topic as understanding of the jet formation, structure 
and propagation thorough magnetosheath and their subsequent impact on the magnetopause will help address magnetospheric response to the dynamic driving by the solar wind and may be even relevant for the long-standing "internal" vs "external" substormtriggering debate. The authors have compiled an extensive data base for addressing this topic using MMS data and I think the paper is suitable for publication after some revisions. I also think that the manuscript would strongly benefit from some additional analysis before clear conclusions can be made. I would recommend the authors to perform some additional analysis (which should not take too long) and address the following in the revised manuscript:

Main comments:

1. The superposed epoch analysis uses normalized time and organizes the data on pre- and post jet intervals based on this time. However, the number of single spacecraft measurements do not provide information on the 3-D jet structure, magnetosheath structure or how the spacecraft might have crossed the jet. Because the spacecraft are nearly stationary when compared to jet propagation speed, the "pre jet" and "post jet" time intervals may correspond to the vastly different spatial regions with respect to jets due to different 3-D field geometries that can arise due various factors, e.g., how spacecraft crosses the jet, the distance to the jet formation region (is the jet accelerating, moving at constant speed or decelerating), how does the jet dynamic pressure relate to the ambient magnetic field pressure in the magnetosheath, to the distance to the magnetopause and due to different spacecraft z-component in GSM coordinates. Note that during 2015-2017, close to the spring and fall equinox times, the MMS GSM z-coordinate in the dayside magnetosheath can be substantial so this list will likely include several high-latitude magnetosheath observations close the dayside high-latitude magnetopause.

I would recommend the authors perform and address the following in the revised manuscript: 
a) Sort all of the identified jet intervals based on the distance to the model magnetopause and model bow shock calculated using prevailing solar wind condition during the jet observations.

b) Show a distribution of the MMS z-position during jet observations and study the dependence of the MMS z-coordinate and distance to the magnetopause and bow shock on the deviation of the "pre jet" and "post jet" angles. Study how the ratio of the local dynamic jet pressure and the pre-jet magnetic field pressure varies as function of distance between magnetopause and bow shock and how this affects the angles.

c) The analysis uses the velocity vector as measured by the MMS to calculate the angles for pre-jet, jet and post jet intervals to address what is the effect of the magnetic field-alignment along the jet. This makes an assumption that the jet is moving along the direction of the ion velocity during the interval identified as a jet. Authors should demonstrate how accurate this assumption is for few cases. They may consider the following:

a) Is there a goof de Hoffman teller frame for the jet structure? If there is not, why not, for example is the jet still accelerating when MMS crosses it? b) If there exists a good de Hoffman teller frame, how does the direction of the Hoffman teller frame velocity of the jet structure compare to the direction of the ion velocity? c) Are the any cases where the 4 spacecraft measurements can be used to determine the actual propagation direction of the jet as supposed to using the measured ion velocity?

These questions are relevant as for example in the simulations of Karimabadi et al., 2014 the direction of the jet motion appeared not to always align with the ion velocity but even sunward flows were seen adjacent to jet structures moving toward magnetopause. Did you observe any sunward flows in your statistics?

d) The pre-state of the magnetosheath field and plasma before the jet formation is likely to be very important for the subsequent jet propagation dynamics. It would be interesting to sort (for example using both IMF clock and cone angle) the jet events

Interactive

comment
Printer-friendly version

Discussion paper

3 
based on the pre-IMF orientation before the radial turning. The current method of taking few minutes of the data before the jet may not be the truly "pre -state" of the magnetosheath depending on the shock geometry and how far the spacecraft is from the shock.

Minor comments:

Lines 10-11: Authors may consider citing recent paper by Nykyri et al., JGR, 2019 which discussed 14 spacecraft observations and the jet impact on substorm onset and showed that magnetosheath jets were associated with bursts of negative $\mathrm{Bz}$ in the magentosheath while IMF was northward. The DMSP spacecraft detected southwardlike IMF erosion of the dayside magnetopause during jet observations during northward IMF, supporting evidence for jet-produced dayside reconnection.

Interactive comment on Ann. Geophys. Discuss., https://doi.org/10.5194/angeo-2019-92, 2019. 